\title{
Spectral Representation for Continuous State Branching Processes
}

By

\section{Yukio OgurA*}

\section{Introduction}

The spectral representation for Galton-Watson processes was given by Karlin and McGregor [4] [5] [6]. Similar results for 'one-dimensional' continuous state branching processes were obtained in Ogura [9]. The object of this article is to give the similar representation for a class of 'multi-dimensional' continuous state branching processes. It is worth to note that our class contains a process with the generator

$$
\sum_{i=1}^{d} x^{i} a^{i} \partial^{2} /\left(\partial x^{i}\right)^{2}+\sum_{i, j=1}^{d} x^{i} b_{j}^{i} \partial / \partial x^{j}-\sum_{i=1}^{d} x^{i} c^{i} \quad\left(a^{i}, c^{i} \geqq 0, \operatorname{det}\left[b_{j}^{i}\right] \neq 0\right)
$$

as a special case.

A continuous state branching process $X=\left(x_{t}, P_{x}\right)$ is a stochastically continuous Markov process on $R_{+}^{d} \cup\{\Delta\} \quad\left(R_{+}=[0, \infty)\right.$ and $\Delta$ is an extra point) with $\Delta$ as a trap, satisfying

$$
E_{x}\left[f_{\lambda}\left(x_{t}\right)\right]=f_{\psi_{t}(\lambda)}(x), \quad x \in R_{+}^{d}, \quad t \geqq 0, \quad \lambda \in R_{+}^{d},
$$

for some $\psi_{t}(\lambda)=\left(\psi_{t}^{1}(\lambda), \ldots, \psi_{t}^{d}(\lambda)\right) \in R_{+}^{d}$, where $f_{\lambda}(x)=e^{-\lambda \cdot x}$ and $f_{\lambda}(\Delta)=0$. We define the infinitesimal cumulant generating fucntion $h^{i}(\lambda)$ by $h^{i}(\lambda)=$ $\dot{\psi}_{0+}^{i}(\lambda)$, and the matrices $H\left(\lambda_{1}, \ldots, \lambda_{d}\right)$ and $H(\lambda)$, for each $\lambda_{1}, \ldots, \lambda_{d}, \lambda \in$ $R_{+}^{d}$, by $H\left(\lambda_{1}, \ldots, \lambda_{d}\right)=\left[h_{j}^{i}\left(\lambda_{i}\right)\right]_{i, j=1}^{d}$ and $H(\lambda)=H(\lambda, \ldots, \lambda)$, where $h_{j}^{i}(\lambda)=$ $\partial h^{i}(\lambda) / \partial \lambda^{j}$. We assume throughout in this paper: (A.1) For some $\lambda_{1}, \ldots$, $\lambda_{d} \in R_{+}^{d}$, all the real parts of the characteristic roots of $H\left(\lambda_{1}, \ldots, \lambda_{d}\right)$ are negative. Then we can see that there exists a maximum element

Communicated by H. Yoshizawa, September 6, 1973.

* Department of Mathematics, Saga University, Saga. 
$\gamma$ in the zero points of $h(\lambda)$ (Lemma 3.2). Our next assumptions are: (A.2) Each $h^{i}(\lambda)$ is analytic at $\gamma$. (A.3) The real parts of the characteristic roots $\mu_{1}, \ldots, \mu_{d}$ of the matrix $H(\gamma)$ are negative.

Under the assumptions above we can choose an appropriate vector $\gamma_{0}<\gamma$ satisfying $(2.1) \sim(2.4)$ in Lemma 2.1 below. ${ }^{1)}$ Now we let $\mathscr{A}$ be the space of all (complex valued and real) analytic functions in $\lambda>\gamma_{0}$, and define the linear operator $\mathfrak{D}$ on it by

$$
\mathfrak{D} u(\lambda)=\sum_{i=1}^{d} h^{i}(\lambda) u_{i}(\lambda), \quad u(\lambda) \in \mathscr{A}
$$

Let $\mathscr{C}_{0}$ be the Banach space of all continuous functions $\phi(x)$ in $x \in R_{+}^{d}$ with the finite norm $\|\phi(x)\|=\sup \left|e^{\gamma_{0} \cdot x} \phi(x)\right|$ and satisfying $\lim _{x \rightarrow \infty} e^{\gamma_{0} \cdot x} \phi(x)$ $=0$. $\mathscr{P}$ is the subset of $\mathscr{C}_{0}$ consists of the functions with the form of $\phi(x)=e^{-\gamma \cdot x} \times\left(\right.$ a polynomial in $\left.x^{1}, \ldots, x^{d}\right)$. Then the semigroup $T_{t}$ of our continuous state branching process is positive and contractive on $\mathscr{C}_{0}$. We denote the infinitesimal generator by $\mathscr{G}$, and its restriction to $\mathscr{P}$ by $\mathfrak{G}_{0}$. Finally we put $\mu_{\alpha}=\sum_{i=1}^{d} \alpha^{i} \mu_{i}$ for $\alpha=\left(\alpha^{1}, \ldots, \alpha^{d}\right) \in Z_{+}^{d}\left(Z_{+}=\{0,1\right.$, $2, \ldots\})$ and let $\left\{v_{0}, v_{1}, \ldots\right\}$ be the set of all distinct elements of $\left\{\mu_{\alpha} ; \alpha \in\right.$ $\left.Z_{+}^{d}\right\}$. Also we set $R(v)=\left\{\alpha \in Z_{+}^{d} ; \mu_{\alpha}=v\right\}$ and $R_{k}=R\left(v_{k}\right)$. Throughout in the following three theorems we assume (A.1) (A.3).

Theorem 1. The set of all eigenvalues of $\mathfrak{D}$ is equal to $\left\{v_{0}\right.$, $\left.v_{1}, \ldots\right\}$. There are $u^{i}(\lambda) \in \mathscr{A}, i=1, \ldots, d$, with

$$
u^{i}(\gamma)=0, \quad \operatorname{det}\left[u_{j}^{i}(\gamma)\right]_{i, j=1}^{d} \neq 0,
$$

such that each $u^{\alpha}(\lambda)=\prod_{i=1}^{d}\left(u^{i}(\lambda)\right)^{\alpha^{i}}$ is an eigenfunction ${ }^{2)}$ of $\mathfrak{D}$ corresponding to $\mu_{\alpha}$. Further the eigenspace of $\mathfrak{D}$ corresponding to $v_{k}$ is the linear hull of $\left\{u^{\alpha}(\lambda) ; \alpha \in R_{k}\right\}$.

We define the system of functions $\left\{\phi_{\alpha}(x)\right\} \subset \mathscr{P}$ by

$$
\sum_{\alpha \in Z_{+}^{d}} \phi_{\alpha}(x) \zeta^{\alpha}=e^{-x \cdot v(\zeta)}, \quad \zeta \text { is near to } 0,
$$

where $v(\zeta)$ is the inverse function of $u(\lambda)=\left(u^{1}(\lambda), \ldots, u^{d}(\lambda)\right)$ at $\lambda=\gamma[\zeta=0]$,

1) We say that $\lambda \in \boldsymbol{R}^{d}$ is larger [not less] than $\lambda^{\prime} \in \mathbf{R}^{d}$ (and denote $\lambda>\lambda^{\prime}\left[\lambda \geqq \lambda^{\prime}\right]$ ) if all its corresponding com ponents are so. These notions are extended for matrices.

2) We call $\xi \neq 0$ an eigenfunction and $\nu \in C$ the corresponding eigenvalue of a linear operator $L$, if $(L-\nu I)^{n} \xi=0$ for some $n \in Z_{+}$. 
and $\zeta^{\alpha}=\prod_{i=1}^{d}\left(\zeta^{i}\right)^{\alpha^{2}}$

Theorem 2. The set of all eigenvalues of $\mathfrak{5}_{0}$ is equal to $\left\{v_{0}\right.$, $\left.v_{1}, \ldots\right\}$. Each $\phi_{\alpha}(x)$ is an eigenfunction of $\mathfrak{5}_{0}$ corresponding to $\mu_{\alpha}$. Further the eigenspace of $\mathfrak{G}_{0}$ corresponding to $v_{k}$ is the linear hull of $\left\{\phi_{\alpha}(x) ; \alpha \in R_{k}\right\}$.

Theorem 3 (main theorem). The semigroup $T_{t}$ admits the spectral representation

(0.5) $\quad T_{t} f_{\lambda}(x)=\sum_{k=0}^{\infty} e^{v_{k} t} \sum_{\alpha \in R_{k}} \phi_{\alpha}(x)\left\{u^{\alpha}(\lambda)+\sum_{\substack{\beta \in R_{k} \\ \beta<\alpha}} b_{\beta}^{\alpha}(t) u^{\beta}(\lambda)\right\}, t \geqq t_{0}, \lambda \in K, x \in R_{+}^{d}$ for any compact set $K$ in $\left\{\lambda ; \lambda>\gamma_{0}\right\}$, where $t_{0} \geqq 0$ is a constant depending only on $K, b_{\beta}^{\alpha}(t)$ is a polynomial in $t$, and ' $\prec$ ' is the total order defined by (1.4) below. The sum converges absolutely and uniformly on $\left\{t \geqq t_{0}\right\} \times K$.

The proofs of these theorems are given in section 2 . It is reduced to the normalization of the analytic contractive semigroup $\psi_{t}(\lambda)$, which we discuss in sections 1 and 2. In section 3 we shall show that (A.1) implies the existence of $\gamma$ and the nonpositivity of the real parts of characteristic roots of $H(\gamma)$. Section 4 is devoted to two examples and some remarks.

All the principal arguments in this article are also valid for the corresponding class of multitype Galton-Watson processes with continuous time.

\section{Preliminary Lemmas}

The normalization (or the linearization) of an analytic contractive semigroup on $\boldsymbol{R}^{d}$ has been investigated by many mathematicians (e.g. Poincaré [10], Sternberg [12], Karlin and McGregor [6], etc.). Especially the results of [6] seems to give an implicit proof for our case with continuous time. But we will give a different proof which is more direct.

Let $\mathscr{A}(\gamma)$ be the space of all germs of analytic functions at $\gamma^{3)}$. For

3) Each $u \in \mathscr{A}(\gamma)$ is identified with a number series $\left\{u_{\alpha} \equiv \partial^{|\alpha|} u(\gamma) /\left(\partial z^{1}\right)^{\alpha^{1}} \cdots\left(\partial z^{d}\right)^{\alpha^{d}} ; \alpha \in\right.$ $\left.Z_{+}^{d}\right\}$ satisfying $\sum\left|u_{\alpha}\right| \delta^{\alpha}<\infty$ for some $\delta>0\left(\delta \in \boldsymbol{R}^{d}\right)$. We rewrite $u_{e_{j}}$ as $u_{\jmath}$, where $e_{\gamma}$ is the unit vector with $1 j$-th component. Further we also identify a germ $u \in \mathscr{A}(\gamma)$ with its arbitrary element $u(z) \in u$ where no confusions occur. 
$h^{i} \in \mathscr{A}(\gamma), i=1, . ., d$, with $h^{i}(\gamma)=0$, we define the linear operator $\mathfrak{D}_{(\gamma)}$ by

$$
\mathfrak{D}_{(\gamma)}=\sum_{i=1}^{d} h^{i} \hat{c} / \partial z^{i}
$$

Lemma 1.1.4) Let the characteristic roots of the Jacobian matrix $H=\left[h_{j}^{i}\right]_{i, j=1}^{d}$ satisfy condition (A.3). Then there exist $u^{i} \in \mathscr{A}(\gamma), \quad i=$ $1, \ldots, d$, satisfying $(0.3)$ and

$$
\mathfrak{D}_{(\vartheta)} u^{i}=\mu_{i} u^{i}+\sum_{\substack{\zeta \in R_{\zeta\left(e_{i}\right)} \\ \zeta<e_{i}}} c_{\zeta}^{i} u^{\zeta}, \quad i=1, \ldots, d
$$

where $c_{\zeta}^{i}$ are constants.

Proof. 1) By a linear change of variables, we may assume

$$
\left.H=\left|\begin{array}{lll}
H_{1} & & 0 \\
& \ddots & \\
0 & & H_{m}
\end{array}\right|, \quad H_{j}=\mid \begin{array}{ccc}
\mu_{j}^{\prime} & & 0 \\
\varepsilon & \ddots & \\
\ddots & \ddots & \ddots \\
0 & \varepsilon & \mu_{j}^{\prime}
\end{array}\right], \quad j=1, \ldots, m,
$$

where $H_{j}$ is a $d_{j} \times d_{j}$ matrix with $d_{j} \geqq 1 \quad\left(\sum_{j=1}^{m} d_{j}=d\right), \mu_{1}^{\prime}, \ldots, \mu_{m}^{\prime}$ satisfy

$$
0>\operatorname{Re} \mu_{1}^{\prime} \geqq \cdots \geqq \operatorname{Re} \mu_{m}^{\prime},
$$

and $\varepsilon>0$ is an arbitrary constant determined below. In fact, for the original Jacobian matrix $H$ we can find a regular matrix $Q$ such that $Q^{-1} H Q$ has the form of (1.2). Setting $\tilde{h}^{i}(z)=\sum_{j=1}^{d} q_{i j}^{-1} h^{j}\left(z^{t} Q\right)$ and $\widetilde{\mathfrak{D}}_{(\gamma)}=$ $\sum_{i=1}^{d} \tilde{h}^{i} \partial / \partial z^{i}$, where $q_{i j}^{-1}$ is the $(i, j)$-component of the inverse matrix $Q^{-1}$ of $Q$, we have

$$
\widetilde{\mathfrak{D}}_{(\gamma)} \tilde{u}(z)=\mathfrak{D}_{(\gamma)} u\left(z^{t} Q\right),
$$

for $\tilde{u}(z)=u\left(z^{t} Q\right)$. Hence, if $\tilde{u}^{1}(z), \ldots, \tilde{u}^{d}(z)$ satisfy (0.3) and (1.1) with respect to $\widetilde{\mathfrak{D}}_{(\gamma)}$, then $u^{1}(z)=\tilde{u}^{1}\left(z^{t} Q^{-1}\right), \ldots, u^{d}(z)=\tilde{u}^{d}\left(z^{t} Q^{-1}\right)$ also satisfy (0.3) and (1.1).

2) Now we can define the total order ' $\prec$ ' in $Z_{+}^{d}$ by

$$
\alpha \prec \beta \quad \text { if }|\alpha|>|\beta| \text {, or }|\alpha|=|\beta| \text { and } \alpha^{i_{0}}<\beta^{i_{0}} \text {, }
$$

4) This lemma is still valid if condition (A.3) is replaced by $\mu_{1}, \ldots, \mu_{d}$ laying inside of a convex cone in the Gauss plane with the vertex 0 . 
where $|\alpha|=\alpha^{1}+\cdots+\alpha^{d}$ and $i_{0}=\max \left\{i ; \alpha^{i} \neq \beta^{i}\right\}$. Further let $T=\{1, \ldots, d\}$, $T_{j}=\left\{i \in T ; 1 \leqq i-\left(d_{1}+\cdots+d_{j-1}\right) \leqq d_{j}\right\}^{5)}, T_{j}^{0}=\left\{d_{1}+\cdots+d_{j-1}+1\right\}, T_{j}^{1}=T_{j}-$ $T_{j}^{0}, \quad T^{0}=\sum_{j=1}^{m} T_{j}^{0}$ and $T^{1}=\sum_{j=1}^{m} T_{j}^{1}$. We may assume $\mu_{i}=\mu_{j}^{\prime}$ for $i \in T_{j}$ without any loss of generality. Here we define the semiorder ' $\prec_{T}$ ' in $T$ by

$i \prec_{T} j$ if $\mu_{i} \neq \mu_{j}$ and $\mu_{j}=\mu_{\beta}$ for some $\beta \in Z_{+}^{d}$ with $\beta^{i} \geqq 1$, or $i, j \in T_{k}$ and $i<j$.

Note that if $\zeta \in R\left(\mu_{i}\right)$ and $\zeta \prec e_{i}$, there exist nonnegative integers $\zeta^{j}$, $j \prec_{T} i$ such that

$$
\zeta=\sum_{j<T} \zeta^{j} e_{j}
$$

Since $T$ is a finite set, we can define $S_{1}$ by the set of all its minimal (w.r.t. the semiorder ' $\prec_{T}$ ) ) elements, $S_{2}$ by the set of all minimal elements of $T-S_{1}, \ldots$ Obviously for some $p \leqq d, T=\sum_{k=1}^{p} S_{k}$.

3) We shall show that for each $i \in S_{1}$ there exists $u^{i} \in \mathscr{A}(\gamma)$ such that

$$
\begin{gathered}
\mathfrak{D}_{(\gamma)} u^{i}=\mu_{i} u^{i}, \\
u^{i}(\gamma)=0, \quad u_{j}^{i}=\delta_{i j}, \quad j=1, \ldots, d .
\end{gathered}
$$

Let $u^{i} \equiv\left\{u_{\alpha}^{i}\right\} \in \mathscr{A}(\gamma)$ be a solution of (1.6). Then comparing the expansion coefficients in the both sides, we have

$$
\sum_{j=1}^{d} \sum_{\substack{\alpha+\beta=\eta+e_{j} \\ \alpha, \beta \geqq 0}} \alpha^{j} u_{\alpha}^{i} h_{\beta}^{j}=\mu_{i} u_{\eta}^{i}, \quad \eta \in Z_{+}^{d},
$$

so that by (1.2)

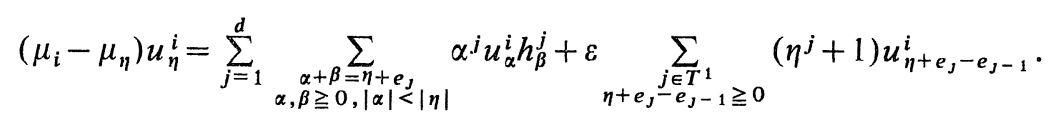

It is clear that $\left\{u_{\eta}^{i},|\eta| \leqq 1\right\}$ defined by (1.7) satisfy (1.8). For $|\eta|>1$, $\mu_{i} \neq \mu_{\eta}$ because of $i \in S_{1}$, and we can determine $\left\{u_{\eta}^{i}\right\}$ inductively and uniquely by (1.8). Now we shall show that $\sum_{\eta \in Z_{+}^{d}}\left|u_{\eta}^{i}\right| \delta^{\eta}<\infty$ for some

5) We make a convention that $d_{1}+\cdots+d_{\jmath-1}=0$ when $j=1$. 
$\delta>0, \delta \in \mathbb{R}^{d}$. By (A.3) we can choose a constant $c>0$ and a positive integer $q$ such that

$$
\left|\mu_{i}-\mu_{\eta}\right| \geqq c|\eta|, \quad|\eta|>q .
$$

Since $h^{j} \in \mathscr{A}(\gamma), j=1, \ldots, d$, we can find constants $L_{1}, K_{1}>0$ such that

$$
\left|h_{\alpha}^{j}\right| \leqq L_{1} K_{1}^{|\alpha|}, \quad \alpha \in Z_{+}^{d}, \quad j=1, \ldots, d .
$$

Set $\left\|u^{i}\right\|_{k}=\max \left\{|\eta|\left|u_{\eta}^{i}\right| ;|\eta|=k\right\}$. Then by (1.8) (1.10) we have

$$
c\left\|u^{i}\right\|_{k} \leqq L_{2} \sum_{l=1}^{k-1}\left\|u^{i}\right\|_{l} K^{k-l}+\varepsilon d\left\|u^{i}\right\|_{k}
$$

for some constants $L_{2}, K>0$. Choosing $\varepsilon>0$ so small that $c-\varepsilon d>0$ and setting $L=L_{2} /(c-\varepsilon d)$, we see

$$
\left\|u^{i}\right\|_{k} \leqq L \sum_{l=1}^{k-1}\left\|u^{i}\right\|_{k} K^{k-l}
$$

Hence, by the induction,

$$
\left\|u^{i}\right\|_{k} \leqq L(K L+K)^{k-q-1} \sum_{l=1}^{q}\|u\|_{l} K^{q+1-l}, \quad k>q
$$

and we obtain the conclusion for a $\delta=\left(\delta^{1}, \ldots, \delta^{d}\right)$ with $0<\delta^{i}<(K L+$ $K)^{-1}, i=1, \ldots, d$.

4) Now we shall prove (1.1) by the induction for the suffix $k$ of $S_{k}$. For $k=1$, the assertion of 3 ) is just the answer. Suppose that we have obtained $u^{j} \in \mathscr{A}(\gamma)$, for each $j \in S_{1}+\cdots+S_{k-1}$, satisfying (1.1) and (1.7), and take an $i \in S_{k}$. Then we can also obtain the germs $u^{\zeta}=$ $\prod_{j=1}^{d}\left(u^{j}\right)^{\zeta^{j}}, \zeta \in R\left(\mu_{i}\right), \zeta \prec e_{i}$ by (1.5). They satisfy

$$
u_{\eta}^{\zeta}= \begin{cases}0, & |\eta| \leqq|\zeta|, \quad \eta \neq \zeta, \\ 1, & \eta=\zeta .\end{cases}
$$

Now let $u^{i} \equiv\left\{u_{\alpha}^{i}\right\} \in \mathscr{A}(\gamma)$ be a solution of (1.1). Comparing the expansion coefficients, we have

$$
\left(\mu_{i}-\mu_{\eta}\right) u_{\eta}^{i}=v_{\eta}^{i}+w_{\eta}^{i}, \quad \eta \in Z_{+}^{d},
$$

where $v_{\eta}^{i}$ is given by the right side of (1.8), and $w_{\eta}^{i}$ by 


$$
w_{\eta}^{i}=-\sum_{\substack{\zeta \in R\left(\mu_{i}\right) \\ \zeta \prec e_{\imath}}} c_{\zeta}^{i} u_{\eta}^{\zeta}
$$

Define $\left\{u_{\eta}^{i} ;|\eta| \leqq 1\right\}$ by (1.7), and $\left\{c_{\eta}^{i} ;|\eta|=1\right\}$ by

$$
c_{e_{l}}^{i}= \begin{cases}\varepsilon, & l=i-1, \quad i \in T^{1}, \\ 0, & \text { otherwise. }\end{cases}
$$

Then obviously (1.12) holds for $|\eta| \leqq 1$. For $|\eta|>1$, note that $\eta \prec e_{i}$ always holds, and by (1.11) and (1.12) it holds

$$
v_{\eta}^{i}-\sum_{\substack{\zeta \in R\left(\mu_{1}\right) \\ \zeta<e_{i},|\zeta|^{\prime}<|\eta|}} c_{\zeta}^{i} u_{\eta}^{\zeta}-c_{\eta}^{i}=0, \quad \eta \in R\left(\mu_{i}\right)
$$

Hence, setting say $u_{\eta}^{i}=0$ for $\eta \in R\left(\mu_{i}\right),|\eta|>1$, we obtain $\left\{u_{\eta}^{i}\right\}$ and $\left\{c_{\eta}^{i}\right.$; $\left.\eta \prec e_{i}\right\}$ inductively and uniquely.

To show that $\sum\left|u_{\eta}^{i}\right| \delta^{\eta}<\infty$ for some $\delta>0$, we note $w^{i} \equiv \sum c_{\zeta}^{i} u^{\zeta} \in$ $\mathscr{A}(\gamma)$, so that it holds for some $L_{1}^{\prime}, K^{\prime}>0$

$$
\left|w_{\eta}^{i}\right| \leqq L_{1}^{\prime} K^{\prime}|\eta|, \quad \eta \in Z_{+}^{d} .
$$

Choosing a sufficiently small $\varepsilon>0$, and setting $L=L^{\prime} /(c-\varepsilon d)$, we see as before that

$$
\left\|u^{i}\right\|_{k} \leqq L \sum_{l=1}^{h-1}\left\|u^{i}\right\|_{l} K^{k-l}+L^{\prime} K^{\prime k}, \quad k>q
$$

Hence by the induction it follows

$$
\begin{aligned}
& \left\|u^{i}\right\|_{k} \leqq L(L K+K)^{k-q-1} \sum_{l=1}^{q}\left\|u^{i}\right\|_{l} K^{q+1-l}+L^{\prime} K^{\prime k} \\
& +L L^{\prime} K \sum_{j=0}^{k-q-2}(L K+K)^{j} K^{\prime k-j-1}
\end{aligned}
$$

and we obtain the conclusion for a $\delta=\left(\delta^{1}, \ldots, \delta^{d}\right)$ with $0<\delta^{i}<((L K+$ $\left.K) \vee K^{\prime}\right)^{-1}$.

Q.E.D.

The next lemma will be also used in section 2 .

Lemma 1.2. Let $u^{i} \in \mathscr{A}(\gamma), \quad i=1, \ldots, d$, satisfy (0.3). Then; 1) If $\xi=\left\{\xi_{\alpha}\right\} \in \mathscr{A}(0)$ and $\sum_{\alpha} \xi_{\alpha} u^{\alpha}=0$ then $\xi=0$. 2) For each $w \in \mathscr{A}(\gamma)$ there 
corresponds a $\xi=\left\{\xi_{\alpha}\right\} \in \mathscr{A}(0)$ such that $w=\sum_{\alpha} \xi_{\alpha} u^{\alpha}$. 3) For each $\xi=$ $\left\{\xi_{\alpha}\right\} \in \mathscr{A}(0)$, it holds $\mathfrak{D}_{(\gamma)} \sum_{\alpha} \xi_{\alpha} u^{\alpha}=\sum_{\alpha} \xi_{\alpha} \mathfrak{D}_{(\gamma)} u^{\alpha}$.

Proof. 1) Let $u^{i}(z) \in u^{i}, i=1, \ldots, d$. Then each $u^{i}(z)$ is analytic in a common neighborhood $U$ of $\gamma$. Taking the change of the variables $\zeta=u(z)$, we have

$$
\xi(\zeta)=\sum_{\alpha} \xi_{\alpha} u^{\alpha}(z)=0, \quad \zeta \in u(U)
$$

Hence $\breve{\zeta}=0$ follows.

2) Let $v(\zeta) \equiv\left(v^{1}(\zeta), \ldots, v^{d}(\zeta)\right)$ be the inverse function of $\left(u^{1}(z), \ldots\right.$, $\left.u^{d}(z)\right)$ at $z=\gamma[\zeta=0]$. Then $\xi(\zeta) \equiv w(v(\zeta))$ is analytic at 0 . Taking the germ $\xi \in \mathscr{A}(0)$ containing $\xi(\zeta)$, we have $w=\sum_{\alpha} \xi_{\alpha} u^{\alpha}$.

3) Since $\xi \in \mathscr{A}(0)$, it holds $\sum_{\alpha}\left|\xi_{\alpha}\right| \delta^{\alpha}<\infty$ for some $\delta=\left(\delta^{1}, \ldots, \delta^{d}\right)>0$. By (0.3) we can choose a neighborhood $U_{1} \subset \bar{U}_{1} \subset U$ of $\gamma$ such that $\left|u^{i}(z)\right|<\delta^{i}$ for all $z \in U_{1}$. Hence the series $\sum_{\alpha} \xi_{\alpha} u^{\alpha}(z)$ converges absolutely and uniformly on $U_{1}$. Further, since

$$
\left|\frac{\partial}{\partial z^{j}} u^{\alpha}(z)\right|=\left|\sum_{i=1}^{d} \alpha^{i} u^{\alpha-e_{i}}(z) \frac{\partial}{\partial z^{j}} u^{i}(z)\right|<K \delta^{\alpha}, z \in U_{1},
$$

the series $\sum_{\alpha} \xi_{\alpha} \partial u^{\alpha}(z) / \partial z^{j}$ also converges absolutely and uniformly on $U_{1}$. Hence we have $\partial\left(\sum_{\alpha} \xi_{\alpha} u^{\alpha}(z)\right) / \partial z^{j}=\sum_{\alpha} \xi_{\alpha} \partial u^{\alpha}(z) / \partial z^{j}$, and obtain the conclusion.

\section{Proof of Theorems}

In this section we shall prove Theorems $1 \sim 3$ assuming the following lemma, which we shall show in section 3 .

Lemma 2.1. Let (A.1) (A.3) be satisfied. Then there is a $\gamma_{0}<\gamma$, such that each $h^{i}(\lambda)$ and $\psi_{t}^{i}(\lambda)$ are analytically continued to the domain $\lambda>\gamma_{0}$, and that

$$
\begin{aligned}
& \dot{\psi}_{t}(\lambda)=h\left(\psi_{t}(\lambda)\right), \quad \psi_{0}(\lambda)=\lambda, \quad \lambda>\gamma_{0}, \\
& T_{t} f_{\lambda}(x)=f_{\psi_{t}(\lambda)}(x), \quad x \in R_{+}^{d}, \quad t \geqq 0, \quad \lambda>\gamma_{0}, \\
& \psi_{t}(\lambda)>\gamma_{0}, \quad \lambda>\gamma_{0}, \quad t \geqq 0,
\end{aligned}
$$




$$
\lim _{t \rightarrow \infty} \psi_{t}(\lambda)=\gamma, \quad \lambda>\gamma_{0} .
$$

Let $u^{i}$ be the one obtained in Lemma 1.1 and $u^{\alpha}=\prod_{i=1}^{d}\left(u^{i}\right)^{\alpha}$.

Lemma 2.2. $u^{\alpha}$ is an eigenfunction of $\delta_{(\gamma)}$ corresponding to $\mu_{\alpha}$. There are no other eigenvalues than $v_{0}, v_{1}, \ldots$, and the eigenspace of $\mathfrak{D}_{(\gamma)}$ corresponding to $v_{k}$ is the linear hull of $\left\{u^{\alpha} ; \alpha \in R_{k}\right\}$. Further $\left\{u^{\alpha}\right\}$ satisfies

$$
\mathfrak{I}_{(\gamma)} u^{\alpha}=v_{h}\left\{u^{\alpha}+\sum_{\substack{\beta \in R_{h} \\ \beta<\alpha}} c_{\beta}^{\alpha} u^{\beta}\right\}, \quad \alpha \in R_{h}, \quad k=0,1, \ldots
$$

where $c_{\beta}^{\alpha}$ are constants.

Proof. (2.5) is obvious from (1.1), (1.4) and the derivation property of $\mathfrak{D}_{(\gamma)}$ :

$$
\mathfrak{D}_{(\gamma)} u^{\alpha}=\sum_{i=1}^{d} \alpha^{i} u^{\alpha-c}, D_{(\gamma)} u^{i}
$$

From (2.5) it is shown by the induction that each $u^{\alpha}$ is an eigen-function of $D_{(y)}$ corresponding to $v_{k}$ if $\alpha \in R_{k}$. Now let $v$ be an eigenvalue of $\mathfrak{D}_{(\gamma)}$ and $w \neq 0$ be a corresponding eigenfunction. Then, by Lemma 1.2 2), there exists a $\xi \in \mathscr{A}(0)$ such that $w=\sum_{\alpha} \xi_{\alpha} l^{\alpha}$. Hencc, by Lemma 1.23 ), we have

$$
0=\left(I_{(\gamma)}-v I\right)^{n} w=\sum_{k=0}^{\infty} \sum_{\alpha, \beta \in R_{h}} \xi_{\alpha} a_{\alpha \beta}^{n y} u^{\beta},
$$

where $a_{\alpha \beta}^{n v}$ are constants given by

$$
\left(\mathfrak{D}_{(\gamma)}-v I\right)^{n} u^{\alpha}=\sum_{\beta \in R_{k}} a_{\alpha \beta}^{n v} u^{\beta}, \quad \alpha \in R_{k}, \quad k=0,1, \ldots
$$

Hence, by Lemma 1.21 ),

$$
\sum_{\alpha \in R_{k}} \breve{\zeta}_{\alpha} a_{\alpha \beta}^{n v y}=0, \quad \beta \in R_{k}, \quad k=0,1, \ldots
$$

Since $\operatorname{det}\left[a_{\alpha \beta}^{n \nu}\right]_{\alpha, \beta \in R_{k}} \neq 0$ for $v \neq v_{k}$, it holds $\xi_{\alpha}=0$ for $\alpha \in R_{k}$ with $v_{k} \neq v$, and we obtain the conclusions.

Lemma 2.3. There exist $u^{\alpha}(\lambda) \in \mathscr{A}, u^{\alpha}(\lambda) \in u^{\alpha}$ such that for each 
$\alpha \in R_{k}$ it holds

$$
u^{\alpha}\left(\psi_{t}(\lambda)\right)=e^{v_{k} t}\left\{u^{\alpha}(\lambda)+\sum_{\substack{\beta \in R_{k} \\ \beta<\alpha}} b_{\beta}^{\alpha}(t) u^{\beta}(\lambda)\right\}, \quad \lambda>\gamma_{0}, t \geqq 0,
$$

where $b_{\beta}^{\alpha}(t)$ are polynomials in $t$. Moreover $b_{\beta}^{\alpha}(t)$ satisfy

$$
\begin{gathered}
v_{k} b_{\beta}^{\alpha}(0)+b_{\beta}^{\alpha}(0)=v_{k} c_{\beta}^{\alpha}, \\
e^{v_{k} t}\left\{\zeta^{\alpha}+\sum_{\substack{\beta \in R_{k} \\
\beta<\alpha}} b_{\beta}^{\alpha}(t) \zeta^{\beta}\right\}=f_{t}^{\alpha}(\zeta),
\end{gathered}
$$

where $f_{t}(\zeta)=\left(f_{t}^{1}(\zeta), \ldots, f_{t}^{d}(\zeta)\right)$ is given by

$$
f_{t}^{i}(\zeta)=e^{\mu_{i} t}\left(\zeta^{i}+\sum_{\substack{\beta \in R^{\prime}\left(\mu_{i}\right) \\ \beta<e_{i}}} b_{\beta}^{e_{i}}(t) \zeta^{\beta}\right) .
$$

Proof. Take arbitrary $u^{i}(\lambda) \in u^{i}, i=1, \ldots, d$. Then there is a neighborhood $U \subset\left\{\lambda>\gamma_{0}\right\}$ of $\gamma$ where every $u^{i}(\lambda)$ is analytic. We may assume $\psi_{t}(U) \subset U$ by (2.4). By (2.5) it holds

$$
\mathfrak{D}_{(\gamma)} u^{\alpha}(\lambda)=v_{k}\left\{u^{\alpha}(\lambda)+\sum_{\substack{\beta \in R_{k} \\ \beta<\alpha^{\prime}}} c_{\beta}^{\alpha} u^{\beta}(\lambda)\right\}, \quad \lambda \in U, \quad \alpha \in R_{k} .
$$

On the other hand it follows from (0.2) and (2.1)

$$
\left\{\begin{array}{l}
\frac{d}{d t} u^{\alpha}\left(\psi_{t}(\lambda)\right)=\left(\mathfrak{D}_{(\gamma)} u^{\alpha}\right)\left(\psi_{t}(\lambda)\right), \quad t>0, \\
u^{\alpha}\left(\psi_{0}(\lambda)\right)=u^{\alpha}(\lambda), \quad \lambda \in U .
\end{array}\right.
$$

Combining (2.9) and (2.10) we obtain a system of ordinary differential equations, and solving it inductively w.r.t. the order ' $\prec$ ' we see that (2.6) holds for $\lambda \in U$.

To show (2.6) for $\lambda>\gamma_{0}$ we must only see that $u^{\alpha}(\lambda)$ is analytically continued to $\lambda>\gamma_{0}$. For this purpose we put $U_{t}=\psi_{t}^{-1}(U)$. Then $U_{t}$ is a domain including $U$ and $\underset{t>0}{\cup} U_{t} \supset\left\{\lambda>\gamma_{0}\right\}$ by means of (2.4). We use the induction again: suppose that the last sum in the right side of (2.6) is analytic in $\lambda>\gamma_{0}$ (this is trivially valid when $\alpha$ is minimal w.r.t. ' $\prec$ '). Then, since the left side of (2.6) can be analytically continued to $U_{t}$ for each $t>0, u^{\alpha}(\lambda)$ can also be so. Hence we obtain the conclusion by the uniqueness property of the analytic continuation. 
(2.7) follows from equations (2.6), (2.9), (2.10) and the uniqueness of expansion coefficients w.r.t. $\left\{u^{\alpha}(\lambda)\right\}$ which is verified from Lemma 1.2 1). Similarly (2.8) is clear from (2.6).

Proof of Theorem 1. Let $u^{i}(\lambda)=u^{e_{i}}(\lambda) \in \mathscr{A}$ be the ones obtained in Lemma 2.3. Then obviously they satisfy $(0.3)$ and $u^{\alpha}(\lambda)=\prod_{i=1}^{d}\left(u^{i}(\lambda)\right)^{\alpha^{i}}$, $\alpha \in Z_{+}^{d}$, satisfy (2.6). Differentiating (2.6) in $t$ we obtain

$$
\mathfrak{D} u^{\alpha}(\lambda)=v_{k}\left\{u^{\alpha}(\lambda)+\sum_{\substack{\beta \in R_{k} \\ \beta<\alpha}} c_{\beta}^{\alpha} u^{\beta}(\lambda)\right\}, \quad \alpha \in R_{k} .
$$

Hence we see that $u^{\alpha}(\lambda)$ is an eigenfunction of $\mathfrak{D}$ corresponding to $\mu_{\alpha}$. The remaining assertions are clear from Lemma 2.2, since $u \in \mathscr{A}(\gamma)$ is an eigenfunction of $\mathfrak{D}_{(\gamma)}$ corresponding to $v$ if $u(\lambda) \in \mathscr{A}$ is an eigenfunction of $\mathfrak{D}$ corresponding to $v$ and $u(\lambda) \in u$.

Q.E.D.

Let $v(\zeta)=\left(v^{1}(\zeta), \ldots, v^{d}(\zeta)\right)$ be the inverse function of $u(\lambda)=\left(u^{1}(\lambda), \ldots\right.$, $\left.u^{d}(\lambda)\right)$ at $\zeta=0$, and $V$ be a neighborhood of 0 where every $v^{i}(\zeta)$ is analytic. Note that by (2.6) and (2.8) it holds

$$
\psi_{t}(\lambda)=v\left(f_{t}(u(\lambda)) \text { for all } t \geqq 0, \lambda>\gamma_{0} \text { such that } u\left(\psi_{t}(\lambda)\right) \in V\right. \text {. }
$$

Let also $\phi_{\beta}(x)$ be the ones defined by $(0.4)$.

Lemma 2.4. 1) Each $\phi_{\beta}(x)$ belongs to $\mathscr{P}$. More precisely $p_{\beta}(x) \equiv$ $e^{\gamma \cdot x} \phi_{\beta}(x)$ is a polynomial in $x,{ }^{1} \ldots, x^{d}$ of degree $\left.|\beta| .2\right) \phi_{\beta}(x), \beta \in Z_{+}^{d}$, are linearly independent, and their linear hull coincides with $\mathscr{P}$. 3) It holds, for each $\beta \in R_{k}, k=0,1, \ldots$, that

$$
T_{t} \phi_{\beta}(x)=e^{v_{k} t}\left\{\phi_{\beta}(x)+\sum_{\substack{\alpha \in R_{k} \\ \beta<\alpha}} \phi_{\alpha}(x) b_{\beta}^{\alpha}(t)\right\}, \quad x \in S, t \geqq 0
$$

Proof. 1) Set $\check{v}_{\alpha}(x)=\sum_{i=1}^{d} x^{i} v_{\alpha}^{i}$. Then, since

$$
x \cdot v(\zeta)=\gamma \cdot x+\sum_{|\alpha| \geqq 1} \check{v}_{\alpha}(x) \zeta^{\alpha}
$$

we see

$$
e^{-x \cdot v(\zeta)}=e^{-\gamma \cdot x} \sum_{\beta} \sum_{n \leqq|\beta|} \frac{(-1)^{n}}{n !} \sum_{\substack{\alpha_{1}+\cdots+\alpha_{n}=\beta \\\left|\alpha_{i}\right| \geqq 1}} \check{v}_{\alpha_{1}}(x) \cdots \check{v}_{\alpha_{n}}(x) \zeta^{\beta} .
$$


Hence we obtain the conclusion with

$$
p_{\beta}(x)=\sum_{n \leqq|\beta|} \frac{(-1)^{n}}{n !} \sum_{\substack{\alpha_{1}+\ldots+\alpha_{n}=\beta \\\left|\alpha_{i}\right| \geqq 1}} \check{v}_{\alpha_{1}}(x) \cdots \check{v}_{\alpha_{n}}(x) .
$$

2) Let $q_{\beta}(x)$ be the highest degree part of the function $p_{\beta}(x)$. Then by (2.13)

$$
q_{\beta}(x)=\frac{(-1)^{|\beta|}}{|\beta| !}\left(\begin{array}{c}
|\beta| \\
\beta^{1} \ldots \beta^{d}
\end{array}\right) \prod_{i=1}^{d} \check{v}_{e_{i}}(x)^{\beta^{i}}, \quad \beta=\left(\beta^{1}, \ldots, \beta^{d}\right) .
$$

Since $x^{j}=\sum_{i=1}^{d} u_{j}^{i} \check{v}_{e_{i}}(x), j=1, \ldots, d$, the linear combinations of $p_{\beta}(x)$, $|\beta| \leqq n$, span the space of all polynomials of degree not larger than $n$, whose dimension coincides with $\#\{\beta ;|\beta| \leqq n\}$. Hence $p_{\beta}(x),|\beta| \leqq n$, are linearly independent. Since $n$ is arbitrary and $\phi_{\beta}(x)=e^{-\gamma \cdot x} p_{\beta}(x)$, the conclusions are clear.

3) Fix a $t \geqq 0$. Since $v(\zeta), u(\lambda)$ and $\psi_{t}(\lambda)$ are continuous we can find, by means of (2.11), a neighborhood $V_{t} \subset V$ of 0 such that

$$
\psi_{t}(v(\zeta))=v\left(f_{t}(\zeta)\right), \quad \zeta \in V_{t},
$$

and $v\left(V_{t}\right) \subset\left\{\lambda>\gamma_{0}\right\}$. Hence, appealing to (2.2), (2.3), (0.4) and (2.8), we see

$$
T_{t} f_{v(\zeta)}(x)=\sum_{k=0}^{\infty} e^{v_{k} t} \sum_{\beta \in R_{k}}\left\{\phi_{\beta}(x)+\sum_{\substack{\alpha \in R_{k} \\ \beta<\alpha}} \phi_{\alpha}(x) b_{\beta}^{\alpha}(t)\right\} \zeta^{\beta}, \quad \zeta \in V_{r} .
$$

Now, by the uniqueness property of the expansion coefficients of $\zeta^{\beta}$, it is enough to show that

$$
T_{t} f_{v(\zeta)}(x)=\sum_{\beta} T_{t} \phi_{\beta}(x) \zeta^{\beta}, \quad \zeta \text { is near to } 0
$$

But this is clear if we note that

$$
\sum_{\beta}\left|\phi_{\beta}(y) \zeta^{\beta}\right| \leqq e^{-\gamma \cdot y} e^{\tilde{v}(\zeta) \cdot y}
$$

where $\tilde{v}(\zeta)=\left(\tilde{v}^{1}(\zeta), \ldots, \tilde{v}^{d}(\zeta)\right), \quad \tilde{v}^{i}(\zeta)=\sum_{|\alpha| \geqq 1}\left|v_{\alpha}^{i} \zeta^{\alpha}\right|$, whence with the aid of (2.3) it follows

$$
T_{t} \sum_{\beta}\left|\phi_{\beta}(\cdot) \zeta^{\beta}\right|(x)<\infty, \quad \zeta \text { is near to } 0
$$


Proof of Theorem 2. By (2.12) and (2.7), it holds

$$
\left(5_{0} \phi_{\beta}(x)=v_{k}\left\{\phi_{\beta}(x)+\sum_{\substack{\alpha \in R_{k} \\ \beta<\alpha}} c_{\beta}^{\alpha} \phi_{\alpha}(x)\right\}, \quad \beta \in R_{h}, \quad k=0,1, \ldots\right.
$$

Hence we see by the induction that $\phi_{\beta}(x)$ is an eigenfunction of $\mathbf{6 5}_{0}$ corresponding to $\mu_{\beta}$. Other assertions are easily obtained from Lemma 2.4 2) and (2.17) by the similar arguments as in the proof of Lemma 2.2 .

Proof of Theorem 3. Take a compact set $K \subset\left\{\lambda>\gamma_{0}\right\}$. Then by (2.4) we can find a $t_{1}>0$ such that $u\left(\psi_{t}(\lambda)\right) \in V$ for all $\lambda \in K$ and $t \geqq t_{1}$. Hence (2.11) and (0.4) imply

$$
f_{\psi_{t}(\lambda)}(x)=e^{-x \cdot v\left(f_{t}(u(\lambda))\right.}=\sum_{\alpha} \phi_{\alpha}(x) f_{t}^{\alpha}(u(\lambda)), \quad t \geqq t_{1}, \quad \lambda \in K, \quad x \in R_{+}^{d},
$$

and we obtain (0.5) with any $t_{0} \geqq t_{1}$ from (2.8). Now we shall show the absolute and uniform convergence of (0.5). Since the power series $\sum_{\alpha} \phi_{\alpha}(x) \zeta^{\alpha}$ converges on $V$, it converges absolutely and uniformly on a neighborhood $V_{1} \subset V$ of 0 . On the other hand, since $u^{i}(\lambda) \in \mathscr{A}$ and $K \subset$ $\left\{\lambda>\gamma_{0}\right\}$, it is clear $\left|u^{i}(\lambda)\right| \leqq M_{1}, \lambda \in K$. Further, by means of (A.3) we can find a $t_{0} \geqq t_{1}$ such that $\tilde{f}_{t}(\zeta) \equiv\left(\tilde{f}_{t}^{1}(\zeta) \ldots \tilde{f}_{t}^{d}(\zeta)\right) \in V_{1}$ for each $t \geqq t_{0}$ and $\left|\zeta^{i}\right|<M_{1}, i=1, \ldots, d$, where

$$
\tilde{f}_{t}^{i}(\zeta)=\left|e^{\mu_{i} t}\right|\left(\left|\zeta^{i}\right|+\sum\left|b_{\beta}^{i}(t) \zeta^{\beta}\right|\right) .
$$

Combining these facts we have the conclusion.

q.e.d.

Remark 2.1. If $P_{e_{i}}\left(x_{t}=0\right)>0, i=1, \ldots, d$, for some $t \geqq 0$, then for each $\gamma_{1}>\gamma_{0}$ there exists a $t_{0} \geqq 0$ such that $(0.5)$ holds for $t \geqq t_{0}$ and $\lambda \geqq$ $\gamma_{1}$. Indeed in this case $\psi_{t}(\infty)<\infty$, and we can choose a $t_{0} \geqq 0$ such that $u\left(\psi_{t}(\lambda)\right) \in V$ for all $\lambda \geqq \gamma_{1}$ and $t \geqq t_{0}$.

Remark 2.2. If one wants to calculate $\phi_{\beta}(x)$ directly not via $u(\lambda)$, the next formula is useful:

$$
\mathfrak{G} p_{\eta}^{0}(x)=\prod_{i, j=1}^{d} h_{j}^{i} \eta^{j} p_{\eta+e_{i}-e_{j}}^{0}(x)-\sum_{i=1}^{d} \sum_{\substack{\alpha+\beta=n \\|\beta|<|\eta|-1}} \frac{\eta !}{\beta !}(-1)^{|\alpha|} h_{\alpha}^{i} p_{\beta+e_{i}}^{0}(x),
$$

where $p_{\eta}^{0}(x)=\prod_{i=1}^{d}\left(x^{i}\right)^{\eta^{i}}$ and $\eta !=\left(\eta^{1} !\right) \ldots\left(\eta^{d} !\right)$ (cf. Karlin and McGregor 
[6]). But we do not discuss on this further.

\section{Some Properties on a $\mathrm{CBP}$}

The object of this section is to prove Lemmas 3.2 and 2.1. We begin with summarizing some properties on a CBP maintained by $\mathrm{S}$. Watanabe [13]. The infinitesimal cumulant generating function $h^{i}(\lambda)$ has a form of

$$
h^{i}(\lambda)=-a^{i}\left(\lambda^{i}\right)^{2}+\sum_{j=1}^{d} b_{j}^{i} \lambda^{j}+c^{i}-\int_{R_{+}^{d}}\left(e^{-\lambda \cdot y}-1+y^{i} \lambda^{i} I_{\{|y|<1\}}\right) n^{i}(d y),
$$

where $a^{i}, b_{j}^{i}(i \neq j), \quad c^{i}$ are nonnegative and $b_{i}^{i}$ real constants, and $n^{i}(\cdot)$ is a nonnegative measure on $R_{+}^{d}-\{0\}$ with the ordinary regular condition. Conversely, for the functions $h^{1}(\lambda), \ldots, h^{d}(\lambda)$ of the form of (3.1) there corresponds a unique CBP with the infinitesimal cumulant generating functions $h^{1}(\lambda), \ldots, h^{d}(\lambda)$. Furthermore the $\Psi$-semigroup $\left\{\psi_{t}(\lambda)\right\}$ is differentiable in $t$ and satisfies

$$
\begin{aligned}
& \dot{\psi}_{t}(\lambda)=h\left(\psi_{t}(\lambda)\right), \quad \psi_{0}(\lambda)=\lambda, \lambda>0, \\
& \psi_{t}(\lambda) \geqq 0, \quad \lambda \geqq 0, \quad t \geqq 0 .
\end{aligned}
$$

The next lemma is clear from the Perron-Frobenius theorem and the max-mini principle (cf. Gantmacher [2] Chapter XIII §2).

Lemma 3.1. 1) $A$ real square matrix $A$ with nonnegative nondiagonal elements has a real characteristic root $\rho(A)$ which is largest in all the real parts of its characteristic roots. 2) Let $A$ and $A^{\prime}$ be such square matrices with the same order, and $A \geqq A^{\prime}$. Then $\rho(A) \geqq$ $\rho\left(A^{\prime}\right)$.

We note that (3.1) implies

$$
\left\{\begin{array}{l}
h^{i}(\lambda) \text { is analytic in } \lambda>0, \text { continuous on } R_{+}^{d} \\
h^{i}(0) \geqq 0, \quad i=1, \ldots, d, \\
h_{j}^{i}(\lambda) \geqq 0, \quad i, j=1, \ldots, d, \quad i \neq j, \quad \lambda>0, \\
h_{j k}^{i}(\lambda) \leqq 0, \quad i, j, k=1, \ldots, d, \quad \lambda>0 .
\end{array}\right.
$$


Therefore the matrix valued function $H(\lambda)$ in $\lambda>0$ is monotone nonincreasing and hence, by Lemma 3.1, the limit $\rho(0)=\lim _{\lambda \downarrow 0} \rho(H(\lambda))$ exists admitting the possibility of $\infty$, where $\lambda \downarrow 0$ means that every component $\lambda^{i}$ of $\lambda$ tends to 0 from the right. Set

$$
\rho= \begin{cases}\rho(0), & \text { if } h^{1}(0)=\cdots=h^{d}(0)=0, \\ \infty, & \text { otherwise }\end{cases}
$$

Lemma 3.2. Suppose that $h^{i}(\lambda), i=1, \ldots, d$, satisfy (3.4) and (A.1). Then, a) if $\rho \leqq 0$, the zero points set $\Lambda \equiv\left\{\lambda \in R_{+}^{d} ; h(\lambda)=0\right\}$ consists of only one point 0 . b) If $\rho>0$, the nonzero maximum element $\gamma$ of $\Lambda$ exists and $\rho(H(\gamma)) \leqq 0$.

For the proof of Lemma 3.2 the next lemma is essential:

Lemma 3.3. (Sevastyanov [11] and Kotelyanskii [7]). Let $A$ be a real $d \times d$-matrix with nonnegative nondiagonal elements and $a_{i j}$ be its $(i, j)$-component. Then $\rho(A)<0[\leqq 0]$ holds if and only if

$$
(-1)^{p} \operatorname{det}\left[a_{i_{k} i_{l}}\right]_{k, l=1}^{p}>0 \quad[\text { resp. } \geqq 0]
$$

holds for every $1 \leqq i_{1}<\cdots<i_{p} \leqq d$ and $1 \leqq p \leqq d$. Moreover this is equivalent to

$$
(-1)^{p} \operatorname{det}\left[a_{i j}\right]_{i, j=1}^{p}>0 \quad[\geqq 0], \quad p=1, \ldots, d .
$$

Proof of Lemma 3.2. For $d=1$, note that (A.1) and (3.4) imply $\lim _{\lambda^{1} \rightarrow \infty} h^{1}\left(\lambda^{1}\right)=-\infty$. Then the assertions are clear from Figure 1.

For the proof of the case $d \geqq 2$, we set $\lambda^{i}=\left(\lambda^{1}, \ldots, \lambda^{i}, \ldots, \lambda^{d}\right) \in R_{+}^{d-1}$ for each $\lambda \in R_{+}^{d}$, and $(\lambda, x)^{i}=\left(\lambda^{1}, \ldots, \lambda^{i-1}, x, \lambda^{i}, \ldots\right.$, $\left.\lambda^{d-1}\right) \in R_{+}^{d}$ for each $\lambda \in R_{+}^{d-1}$ and $x \in R_{+}$. Then for each $\lambda \in R_{+}^{d-1}$ we can find an $x \in R_{+}$such that $h^{i}\left((\lambda, x)^{i}\right)<0$. Indeed, by (A.1) and (3.5) there are $\lambda_{0} \in R_{+}^{d-1}$

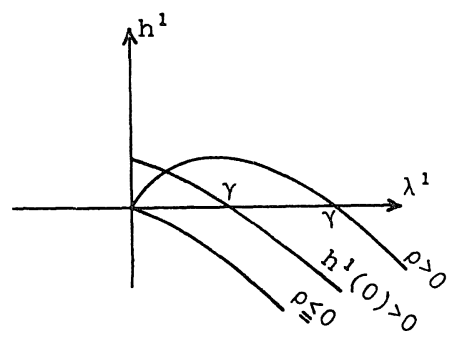

Figure 1. and $x_{0} \in R_{+}$with $h_{i}^{i}\left(\left(\lambda_{0}, x_{0}\right)^{i}\right)<0$. Since $h_{i}^{i}\left(\left(\lambda, x_{0}\right)^{i}\right)$ is nonincreasing, $h_{i}^{i}\left(\left(\lambda, x_{0}\right)^{i}\right)<0$ for $\lambda \geqq \lambda_{0}$. Hence we can find an $x \in R_{+}$satisfying $h^{i}\left((\lambda, x)^{i}\right)<0$ for each $\lambda \geqq \lambda_{0}$ because of 
$h_{i i}^{i}(\cdot) \leqq 0$. Further, for each $\lambda \in R_{+}^{d-1}$, there is a $\mu \geqq \lambda$ with $\mu \geqq \lambda_{0}$. Since $h^{i}\left((\lambda, x)^{i}\right) \leqq h^{i}\left((\mu, x)^{i}\right)$ by (3.4) we have the conclusion. Now by the same arguments as for $d=1$ we can define the function $g^{i}(\lambda)$ by

$$
g^{i}(\lambda)=\max \left\{x \geqq 0 ; h^{i}\left((\lambda, x)^{i}\right)=0\right\},
$$

which can be seen to satisfy

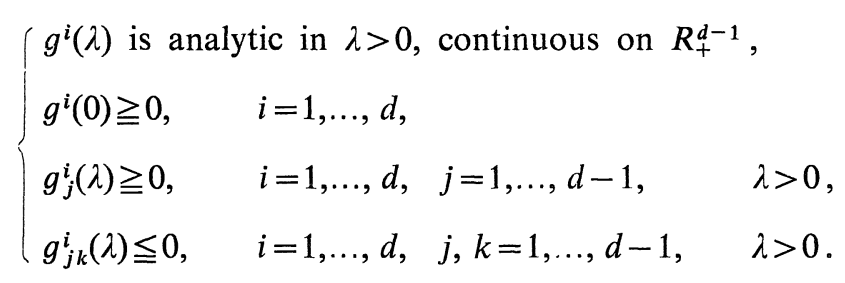

Define the matrices $G\left(\lambda_{1}, \ldots, \lambda_{d}\right)$ for each $\lambda_{1}, \ldots, \lambda_{d}>0\left(\lambda_{i} \in R_{+}^{d-1}\right)$ and $G(\lambda)$ for each $\lambda>0\left(\lambda \in R_{+}^{d}\right)$ by

$$
G\left(\lambda_{1}, \ldots, \lambda_{d}\right)=\left[\begin{array}{cc}
-1 & g_{j-1}^{i}\left(\lambda_{i}\right) \\
\ddots & \ddots \\
g_{j}^{i}\left(\lambda_{i}\right) & -1
\end{array}\right]_{i, j=1}^{d}, \quad G(\lambda)=G\left(\lambda^{1}, \ldots, \lambda^{d}\right) .
$$

Then it can also be seen that (A.1) is equivalent to (A.1) $\quad \rho\left(G\left(\lambda_{1}, \ldots, \lambda_{d}\right)<0\right.$ for some $\lambda_{1}, \ldots, \lambda_{d}>0, \in R_{+}^{d-1}$.

Set $\rho_{1}(0)=\lim _{\lambda \downarrow 0} \rho(G(\lambda))$ and

$$
\rho_{1}= \begin{cases}\rho_{1}(0), & \text { if } g^{1}(0)=\cdots=g^{d}(0)=0, \\ \infty, & \text { otherwise }\end{cases}
$$

Setting also $\Gamma_{i}=\left\{\left(\lambda, g^{i}(\lambda)\right)^{i} ; \lambda \in R_{+}^{d-1}\right\}$, we can rewrite Lemma 3.2 as follows:

Lemma 3.2'. Suppose that $g^{i}(\lambda), i=1, \ldots, d$, satisfy (3.7) and (A.1)'. Then, a) if $\rho_{1} \leqq 0$ the set $\Gamma \equiv \bigcap_{i=1}^{d} \Gamma_{i}$ consists of one point 0 . b) If $\rho_{1}>0$ the nonzero maximum element $\gamma$ of $\Gamma$ exists and $\rho(G(\gamma)) \leqq 0$.

Proof. We shall use the induction. First let $d=2$. When $\rho \leqq 0$, $g^{1}(0)=g^{2}(0)=0$ and the inner normal vectors $n_{1}$ and $n_{2}$ of the curves 


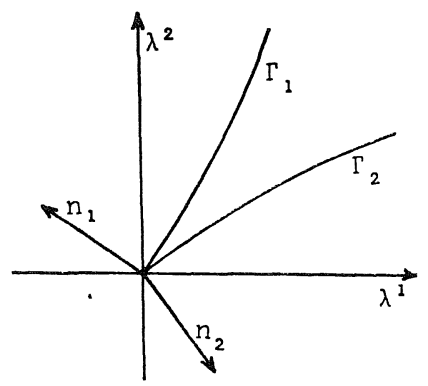

Figure 2. the case of $\rho \leqq 0$.

$\Gamma_{1}$ and $\Gamma_{2}$ at 0 are equal to $(-1$, $\left.\left(g^{1}\right)^{\prime}(0+)\right)$ and $\left(\left(g^{2}\right)^{\prime}(0+),-1\right)$. Hence by (3.5) the base $\left(n_{1}, n_{2}\right)$ has the same orientation as that of $\left(e_{1}, e_{2}\right)$, so that $\Gamma_{1} \cap \Gamma_{2}=\{0=(0,0)\}$ by Figure 2 . When $\rho>0, g^{1}(0)+g^{2}(0)>0$ or the base $\left(n_{1}\right.$, $n_{2}$ ) has the other orientation than that of $\left(e_{1}, e_{2}\right)$. On the other hand by (A.1) $)^{\prime}$ and (3.5) the base $\left(n_{1}^{\prime}, n_{2}^{\prime}\right) \equiv((-1$, $\left.\left.\left(g^{1}\right)^{\prime}\left(\lambda_{1}\right)\right),\left(\left(g^{2}\right)^{\prime}\left(\lambda_{2}\right),-1\right)\right)$ has the same orientation as that of $\left(e_{1}, e_{2}\right)$, so that by Figures 3 and $4 \Gamma_{1} \cap \Gamma_{2}$ contains a point $\gamma$ different from 0 , for which $\rho(G(\gamma)) \leqq 0$.

Now we proceed for the case $d \geqq 3$. First note that, by the similar argument as for the case $d=2$, for each $\lambda \in R_{+}^{d-2}$ and $i=1, \ldots, d-1$ the equation

$$
\left\{\begin{array}{l}
x=g^{i}\left((\lambda, y)^{d-1}\right) \\
y=g^{d}\left((\lambda, x)^{i}\right), \quad(x, y) \geqq 0,
\end{array}\right.
$$

has at most two solutions and, if two, one of them is equal to $0=$ $(0,0)$. Define the vector $\left(g_{i d}^{i}(\lambda), g_{i d}^{d}(\lambda)\right)$ by the solution of (3.8) distinct from 0 if exists, and by 0 otherwise. Setting also $g^{\prime i}(\lambda)=g_{i d}^{i}(\lambda), \quad i=$ $1, \ldots, d-1, \lambda \geqq 0\left(\lambda \in R_{+}^{d-2}\right)$, we can see that 


$$
\left\{\begin{array}{l}
g^{\prime i}(\lambda) \text { is analytic in } \lambda>0, \text { continuous on } R_{+}^{d-2}, \\
g^{\prime i}(0) \geqq 0, \quad i=1, \ldots, d-1, \\
g_{j}^{\prime i}(\lambda) \geqq 0, \quad i=1, \ldots, d-1, \quad j=1, \ldots, d-2, \quad \lambda>0, \\
g_{j k}^{\prime i}(\lambda) \leqq 0, \quad i=1, \ldots, d-1, \quad j, k=1, \ldots, d-2, \quad \lambda>0 .
\end{array}\right.
$$

We define the matrices $G^{\prime}\left(\lambda_{1}, \ldots, \lambda_{d-1}\right)\left(\lambda_{i}>0, \lambda_{i} \in R_{+}^{d-2}\right)$ and $G^{\prime}(\lambda)(\lambda>0$, $\left.\lambda \in R_{+}^{d-1}\right)$, the values $\rho_{1}^{\prime}(0)$ and $\rho_{1}^{\prime}$ and the surfaces $\Gamma_{i}^{\prime}, i=1, \ldots, d-1$ from $g^{\prime i}(\lambda)$ 's as we did from $g^{i}(\lambda)$ 's in the above. Then by virtue of Sylvester's determinant identity (Gantmacher [2] Chapter II) and Lemma 3.3 it can be seen that

$$
\left\{\begin{array}{lll}
\rho_{1} \leqq 0 & \text { implies } g^{d}(0)=0 & \text { and } \rho_{1}^{\prime} \leqq 0 \\
\rho_{1}>0 & \text { implies } g^{d}(0)>0 & \text { or } \rho_{1}^{\prime}>0
\end{array}\right.
$$

$$
\rho\left(G^{\prime}\left(\lambda_{1}, \ldots, \lambda_{d-1}\right)\right)<0 \text { for some } \lambda_{1}, \ldots, \lambda_{d-1}>0, \in R_{+}^{d-2}
$$

Now, by the assumption of the induction, (3.9) and (A.1)" imply the existence of the maximum element $\gamma^{\prime}$ of $\bigcap_{i=1}^{d-1} \Gamma_{i}^{\prime}$. Then $\gamma=\left(\gamma^{\prime}, g^{d}\left(\gamma^{\prime}\right)^{d}\right)$ is the maximum element of $\bigcap_{i=1}^{d} \Gamma_{i}$. If $\rho_{1} \leqq 0$, then $\rho_{1}^{\prime} \leqq 0$, so that $\gamma^{\prime}=0$ by the assumption of the induction. Since $g^{d}(0)=0$ it follows $\gamma=0$ and so $\bigcap_{i=1}^{d} \Gamma_{i}=\{0\}$. Now we consider the case of $\rho_{1}>0$. If $\rho_{1}^{\prime}>0$ then $\gamma^{\prime} \neq 0$, so that $\gamma \neq 0$. If $g^{d}(0)>0$ then $g^{d}\left(\gamma^{\prime}\right)>0$ since $g^{d}(\lambda)$ is monotone nondecreasing, and so $\gamma \neq 0$.

To prove $\rho(G(\gamma)) \leqq 0$ note that the functions $\tilde{g}^{i}(\lambda) \equiv g^{i}\left(\lambda+\gamma^{i}\right)-\gamma^{i}$, $i=1, \ldots, d$, satisfy (3.7), (A.1) and $\tilde{\rho}_{1}(0)=\rho(G(\gamma))$, where $\tilde{\rho}_{1}(0)$ is defined from $\tilde{g}^{i}(\lambda)$ 's as $\rho_{1}(0)$. Now suppose that $\rho(G(\gamma))>0$. Then from what we have proved above it follows the existence $\tilde{\gamma} \neq 0$ in the set $\bigcap_{i=1}^{d} \tilde{\Gamma}_{i}$. But it follows $\gamma+\tilde{\gamma} \in \bigcap_{i=1}^{d} \Gamma_{i}$ from the definition of $\tilde{g}^{i}(\lambda)$, which contradicts the fact that $\gamma$ is maximum in the set $\bigcap_{i=1}^{d} \Gamma_{i}$ Q.E.D.

Remark 3.1. It can also be seen that if $h_{j}^{i}(\lambda)>0, i, j=1, \ldots, d$, $i \neq j$ and $\rho>0$ then $\gamma>0$ and $\rho(H(\gamma))<0$.

Lemma 3.4. If (A.2) and (A.3) are satisfied as well as the assump- 
tions of Lemma 3.2, then there exists a $\gamma_{0}<\gamma$ such that $h^{i}(\lambda)$ is analytic there and $h\left(\gamma_{0}\right)>0$.

Proof. By (A.3) and Lemma $3.3 h_{i}^{i}(\gamma)<0$. Hence there exists a neighborhood $U$ of $\gamma$ such that $h^{i}(\lambda)$ and $g^{i}(\lambda)$ in the proof of Lemma $3.2^{\prime}$ are analytically continued to it and $h^{i}(\lambda)>0$ is equivalent to $\lambda^{i}<$ $g^{i}\left(\lambda^{i}\right)$ on it $\left(\lambda \in R^{d}\right)$. We shall show by the induction the existence of a sequence $\left\{\gamma_{n}\right\} \subset U$ such that $\gamma_{n} \rightarrow \gamma$, $\gamma_{n}<\gamma \quad$ and $\gamma_{n}^{i}<g^{i}\left(\gamma_{n}^{i}\right), \quad i=1, \ldots, d$. Since it is clear for $d=1$ and $d=2$ we assume that it holds for $d-1$. Then, by the same argument as in the proof of Lemma 3.2', there exists a sequence $\left\{\gamma_{n}^{\prime}\right\} \subset R^{d-1}$ such that $\gamma_{n}^{\prime} \rightarrow \gamma^{\prime}, \quad \gamma_{n}^{\prime}<\gamma^{\prime}$ and $\gamma_{n}^{\prime}<g^{\prime i}\left(\gamma_{n}^{\prime i}\right), \quad i=$ $1, \ldots, d-1$, where $\gamma^{\prime}=\gamma^{d} \in R^{d-1}$. By the arguments for $d=2$ we can find a $y_{n i}<g^{d}\left(\gamma_{n}^{\prime}\right) \leqq \gamma^{d}$ satisfying $\gamma_{n}^{\prime i}<$ $g^{i}\left(\left(\gamma_{n}^{\prime i}, x\right)^{d-1}\right)$ for every $y_{n i} \leqq x<$

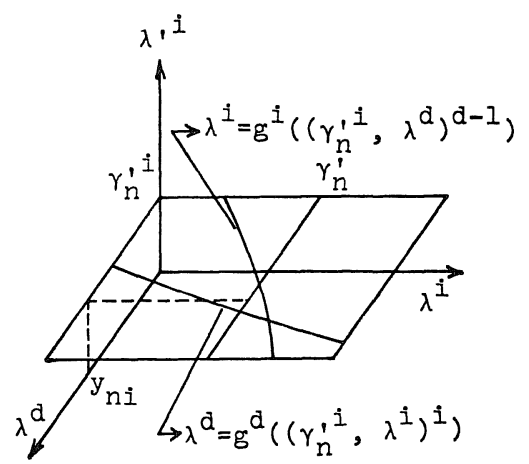

Figure 5. $g^{d}\left(\gamma_{n}^{\prime}\right)$ (cf. Figure 5). Set $y_{n}=\max \left\{y_{n 1}, \ldots, y_{n(d-1)}, g^{d}\left(\gamma_{n}^{\prime}\right)-1 / n\right\}$. Then $\gamma_{n} \equiv$ $\left(\gamma_{n}^{\prime}, y_{n}\right)^{d}$ satisfies the required properties.

Lemma 3.5 (Jirina [3] Theorem 2.2). For a CBP $X=\left(x_{t}, P_{x}\right)$ it holds

$$
\lim _{t \rightarrow \infty} P_{x}\left(x_{t}=0\right)=1, \quad x \in \mathrm{S}
$$

if and only if $\Lambda=\{0\}$.

Corollary 3.1. Condition (A.1) implies

$$
\lim _{t \rightarrow \infty} \psi_{t}(\lambda)=\gamma, \quad \lambda \geqq \gamma
$$

Proof. When $\rho \leqq 0,(3.12)$ is a direct consequence of Lemma 3.2 and (0.1). When $\rho>0$, set $\tilde{h}(\lambda)=h(\lambda+\gamma)$. Then $\tilde{h}(\lambda)$ also has the form of (3.1) and the corresponding $\Psi$-semigroup is $\tilde{\psi}_{t}(\lambda)=\psi_{t}(\lambda+\gamma)-\gamma$. So (3.12) is clear from Lemma 3.2 as in case of $\rho \leqq 0$. 
Finally we shall prove Lemma 2.1.

Proof of Lemma 2.1. By Lemma 3.4 and (3.1) each $h^{i}(\lambda)$ is analytically continued to $\lambda>\gamma_{0}$. Let $\tilde{h}(\lambda)=h\left(\lambda+\gamma_{0}\right)$ and $\tilde{X}$ be the corresponding CBP. Then the corresponding $\Psi$-semigroup $\tilde{\psi}_{t}(\lambda)$ satisfies (3.2) with $\tilde{h}(\lambda)$ instead of $h(\lambda)$ and is analytic in $\lambda>0$. Since the function $\psi_{t}(\lambda+$ $\left.\gamma_{0}\right)-\gamma_{0}\left(\lambda>-\gamma_{0}\right)$ also satisfies the same equation, the uniqueness property of its solution implies

$$
\tilde{\psi}_{t}(\lambda)=\psi_{t}\left(\lambda+\gamma_{0}\right)-\gamma_{0} \quad \lambda>\max \left\{-\gamma_{0}, 0\right\} .
$$

Since the left side of (3.13) is analytic in $\lambda>0$, the right side is analytically continued to there and (3.13) holds for $\lambda>0$ i.e. $\lambda+\gamma_{0}>\gamma_{0}$. Hence $\psi_{t}(\lambda)$ is analytic in $\lambda>\gamma_{0}$ and (2.1) holds. To see (2.2) note that (3.13), (0.1) and the uniqueness theorem of Laplace transforms imply

$$
P(t, x, d y)=e^{-\gamma_{0} \cdot x} \tilde{P}(t, x, d y) e^{\gamma_{0} \cdot y} .
$$

Since $\int \tilde{P}(t, x, d y) e^{-\left(\lambda-\gamma_{0}\right) \cdot y}<\infty$ for each $\lambda>\gamma_{0}$, we have

$$
\int P(t, x, d y) e^{-\lambda \cdot y}=e^{-\gamma_{0} \cdot x} \int \tilde{P}(t, x, d y) e^{-\left(\lambda-\gamma_{0}\right) \cdot y}, \quad \lambda>\gamma_{0} .
$$

But since (3.13) was valid for $\lambda>0$, we obtain (2.2). For (2.3) we have only to note that $(0.1)$ implies $\psi_{t}(\lambda) \geqq \psi_{t}\left(\gamma_{0}\right)$ for $\lambda>\gamma_{0}$ and $\tilde{\psi}_{t}(0)=\psi_{t}\left(\gamma_{0}\right)-$ $\gamma_{0}>0$ holds since $\tilde{\psi}_{t}(0)$ is monotone nondecreasing in $t$ and $\dot{\tilde{\psi}}_{0+}(0)=$ $\tilde{h}(0)=h\left(\gamma_{0}\right)>0$.

Now we shall show (2.4). Since the fixed point $\gamma$ of equation (2.1) is asymptotically stable by (A.3), there exists a neighborhood $U$ of $\gamma$ such that (2.4) holds for $\lambda \in U$ (cf. Coddington and Levinson [1]). Combining this fact with (3.12) we have (2.4) for $\lambda \in U \cup\{\lambda \geqq \gamma\}$. We may assume $\gamma_{0} \in U$ by taking larger $\gamma_{0}<\gamma$ if necessary. Now take a $\lambda>\gamma_{0}$. Then there exist $\lambda_{1}$ and $\lambda_{2}$ in $U \cup\{\lambda \geqq \gamma\}$ such that $\lambda_{1} \leqq \lambda \leqq \lambda_{2}$. Since $\psi_{t}\left(\lambda_{1}\right) \leqq \psi_{t}(\lambda) \leqq \psi_{t}\left(\lambda_{2}\right)$ by $(0.1)$ and $\lim _{t \rightarrow \infty} \psi_{t}\left(\lambda_{1}\right)=\lim _{t \rightarrow \infty} \psi_{t}\left(\lambda_{2}\right)=\gamma$ holds, we obtain (2.4). 


\section{Examples of the Representation}

Example 1 (direct product). Let

$$
\begin{aligned}
h^{i}(\lambda)= & h^{i}\left(\lambda^{i}\right) \\
= & -a_{i}\left(\lambda^{i}\right)^{2}+b_{i}^{i} \lambda^{i}+c_{i} \\
& -\int_{0}^{\infty}\left(e^{-\lambda^{i} y^{i}}-1+y^{i} \lambda^{i} I_{\left\{\left|y^{i}\right|<1\right\}}\right) n^{i}\left(d y^{i}\right), \quad i=1, \ldots, d .
\end{aligned}
$$

This case can be reduced to the results for one-dimensional CBP's, which we now summarize. Assume that $h^{i}\left(\lambda^{i}\right)$ is non-critical and analytic at its maximum zero point $\gamma^{i}$. Then the eigenvalues of $\mathfrak{D}^{i}\left(\mathfrak{D}^{i}\right.$ is the linear operator defined by $\left.\mathfrak{D}^{i} u\left(\lambda^{i}\right)=h^{i}\left(\lambda^{i}\right) u^{\prime}\left(\lambda^{i}\right)\right)$ are $k \mu_{i}, k=0,1, \ldots$, where $\mu_{i}=\left(h^{i}\right)^{\prime}\left(\gamma^{i}\right)$. There exists an eigenfunction $u^{i}\left(\lambda^{i}\right)$ of $\mathfrak{D}^{i}$ corresponding to $\mu_{i}$ satisfying $u^{i}\left(\gamma^{i}\right)=0$ and $\left(u^{i}\right)^{\prime}\left(\gamma^{i}\right) \neq 0$. The function $u^{i}\left(\lambda^{i}\right)^{k}$ is an eigenfunction corresponding to $k \mu_{i}$ and has the inverse Laplace transform $\xi_{k}^{i}\left(d x^{i}\right)$ in the space of signed measures:

$$
u^{i}\left(\lambda^{i}\right)^{h}=\int_{0}^{\infty} e^{-\lambda^{i} x^{i}} \xi_{k}^{i}\left(d x^{i}\right) .
$$

Let $v^{i}\left(\zeta^{i}\right)$ be the inverse function of $u^{i}\left(\lambda^{i}\right)$ at $\lambda^{i}=\gamma^{i}\left[{ }_{\zeta}^{i}=0\right]$. Then the eigenfunction $\phi_{k}^{i}\left(x^{i}\right)$ of $\mathfrak{G}_{0}^{i}$ are given by

$$
e^{-x^{i} v^{1}\left(\zeta^{2}\right)}=\sum_{k=0}^{\infty} \phi_{k}^{i}\left(x^{i}\right)\left(\zeta^{i}\right)^{k}
$$

Now, in our case, conditions (A.1) (A.3) are satisfied where the maximum zero point $\gamma$ is given by $\gamma=\left(\gamma^{1}, \ldots, \gamma^{d}\right)$. It is clear that the set of all eigenvalues of $\mathfrak{D}$ is equal to $\left\{\mu_{\alpha}=\sum_{i=1}^{d} \alpha^{i} \mu_{i} ; \alpha \in Z_{+}^{d}\right\}$ and the corresponding eigenfunction is given by

$$
u^{\alpha}(\lambda)=\prod_{i=1}^{d} u^{i}\left(\lambda^{i}\right)^{\alpha^{2}}, \alpha \in Z_{+}^{d}
$$

The eigenfunctions of $\mathfrak{G}_{0}$ are

$$
\phi_{\alpha}(x)=\prod_{i=1}^{d} \phi_{\alpha^{2}}^{i}\left(x^{i}\right), \quad \alpha \in Z_{+}^{d} .
$$


All the eigenfunctions of $D$ or $\left(\mathfrak{F}_{0}\right.$ are those in the narrow sense, $\left.{ }^{6}\right)$ and the representation $(0.5)$ holds with $b_{\beta}^{\alpha}(t) \equiv 0$. Moreover, in this case, the eigenfunction $u^{\alpha}(\lambda)$ has the inverse Laplace transform

$$
\xi_{\alpha}(d x)=\xi_{\alpha^{1}}^{1}\left(d x^{1}\right) \otimes \cdots \otimes \xi_{\alpha^{d}}^{d}\left(d x^{d}\right), \quad \alpha \in Z_{+}^{d},
$$

where the notation $\otimes$ means the product of the measures. For some classes of $h^{i}\left(\lambda^{i}\right)$ 's we can calculate $\xi_{k}^{i}\left(d x^{i}\right)$ and $\phi_{k}^{i}\left(x^{i}\right)$ precisely (cf. Ogura [9]), so that $\xi_{\alpha}(d x)$ and $\phi_{\alpha}(x)$ by (4.1) and (4.2). Especially, if our CBP is a diffusion, we can see that the transition function has a density w.r.t. the Lebesgue measure, which admits the spectral representation by $\phi_{\alpha}(x)$ only and is symmetric w.r.t. the weight $\exp \left(-\sum_{i=1}^{d} b_{i}^{i} x^{i} / a_{i_{\imath}}\right) d x^{1} \ldots$ $d x^{d} / x^{1} \ldots x^{d}$.

Example 2. Let $d=2$ and

$$
\left\{\begin{array}{l}
h^{1}(\lambda)=-a\left(\lambda^{1}\right)^{2}-b \lambda^{1}, \\
h^{2}(\lambda)=c \lambda^{1}-e \lambda^{2}, \quad a, b, c, e>0 .
\end{array}\right.
$$

In this case $\rho<0$ and $\mu_{1}=-b, \mu_{2}=-e$ are the characteristic roots of the matrix $H$. The eigenfunction of $\mathfrak{D}$ corresponding to $\mu_{1}$ is

$$
u^{1}(\lambda)=\frac{\lambda^{1}}{1+a \lambda^{1} / b}
$$

Here we divide it into two cases:

1) $\mu_{2} \neq m \mu_{1}$ for any $m=1,2, \ldots$ In this case the eigenfunction of D corresponding to $\mu_{2}$ is

$$
u^{2}(\lambda)=\lambda^{2}+\frac{b c}{a} \sum_{n=1}^{\infty}\left(\frac{a}{b} u^{1}(\lambda)\right)^{n} /(n b-e) .
$$

The inverse function $v(\zeta)$ is

$$
v^{1}(\zeta)=\frac{\zeta^{1}}{1-a \zeta^{1} / b}, \quad v^{2}(\zeta)=\zeta^{2}-\frac{b c}{a} \sum_{n=1}^{\infty}\left(\frac{a}{b} \zeta^{1}\right)^{n} /(n b-e)
$$

so that the eigenfunction $\phi_{\alpha}(x)$ of $\mathfrak{F}_{0}$ is given by

6) We call $\xi \neq 0$ an eigenfunction of a linear operator $L$ in the narrow sense if $L \xi=\nu \xi$ holds. 


$$
\begin{aligned}
& \phi_{\alpha}(x)=(-1)^{|\alpha|} \sum_{l=0}^{|\alpha|}\left(\left(\frac{a}{b}\right)^{|\alpha|-l} \mid l !\right) \sum_{p=\left(l-\alpha^{1}\right) \vee \alpha^{2}}^{l}\left(\begin{array}{c}
l \\
p
\end{array}\right)\left(\begin{array}{c}
p \\
\alpha^{2}
\end{array}\right) K(a: l, p) \\
& c^{p-\alpha^{2}}\left(x^{1}\right)^{l-p}\left(x^{2}\right)^{p},
\end{aligned}
$$

where

$$
K(\alpha: l, p)=\sum_{r=p^{-\alpha^{2}}}^{\alpha^{1}-l+p}\left(\begin{array}{c}
p-l \\
\alpha^{1}-l+p-r
\end{array}\right)(-1)^{r} \sum_{\substack{n_{1}+\cdots+n_{p}-\alpha^{2}=r \\
n_{i} \geqq 1}} \prod_{i=1}^{p-\alpha^{2}} \frac{1}{n_{i} b-e} .
$$

They are eigenfunctions in the narrow sense and (0.5) holds with $b_{\beta}^{\alpha}(t) \equiv 0$.

2) $\mu_{2}=m \mu_{1}$ for some $m \geqq 1$. In this case the basic eigenfunction of $D$ corresponding to $\mu_{2}$ is

$$
u^{2}(\lambda)=\lambda^{2}+\frac{b c}{a} \sum_{\substack{n=1 \\ n \neq m}}^{\infty}\left(\frac{a}{b} u^{1}(\lambda)\right)^{n} \frac{1}{n b-e},
$$

and the inverse function $v(\zeta)$ is

$$
v^{1}(\zeta)=\frac{\zeta^{1}}{1-a \zeta^{1} / b}, \quad v^{2}(\zeta)=\zeta^{2}-\frac{b c}{a} \sum_{\substack{n=1 \\ n \neq m}}^{\infty}\left(\frac{a}{b} \zeta^{1}\right)^{n} /(n b-e)
$$

The eigenfunction $\phi_{\alpha}(x)$ of $\mathfrak{6}_{0}$ is same as (4.4) except for that $K(\alpha$; $l, p)$ is replaced by

$$
\tilde{K}(\alpha: l, p)=\sum_{r=p-\alpha^{2}}^{\alpha^{1}-l+p}\left(\begin{array}{c}
p-l \\
\alpha^{1}-l+p-r
\end{array}\right)(-1)^{r} \sum_{\substack{n_{1}+\cdots+n_{p-\alpha}=r \\
n_{1} \geqq 1, n_{l} \neq m}} \prod_{i=1}^{p-\alpha^{2}} \frac{1}{n_{i} b-e} .
$$

The function $u^{2}(\lambda)$ satisfies

$$
\mathfrak{D} u^{2}(\lambda)=\mu_{2} u^{2}(\lambda)+c\left(\frac{a}{b}\right)^{m-1} u^{1}(\lambda)^{m}
$$

and therefore (0.5) holds with

$$
b_{\beta}^{\alpha}(t)= \begin{cases}\left(\begin{array}{l}
\alpha^{2} \\
\beta^{2}
\end{array}\right)(K t)^{\alpha^{2}-\beta^{2}}, & \text { for } \beta^{1}+m \beta^{2}=\alpha^{1}+m \alpha^{2}, 0 \leqq \beta^{2}<\alpha^{2}, \\
0, & \text { othewise, }\end{cases}
$$

where $K=c(a / b)^{m-1}$.

Remark 4.1. For the $h^{2}$ in (4.3), we can calculate the eigenfunctions concretely for somewhat wider classes of $h^{1}(\lambda)=h^{1}\left(\lambda^{1}\right)$; e.g. for the 
classes in the examples in Ogura [9].

Remark 4.2. The case 2) of Example 2 gives an example that the semigroup $T_{t} f_{\lambda}$ is not always diagonalizable even if the matrix $H$ is so.

Remark 4.3. In general the transition function of a CBP is nonsymmetrizable: For example, when a CBP is a diffusion, it is symmetrizable if and only if it is of a direct product case i.e. the case of $b_{j}^{i}=0(i \neq j)$ (cf. Nelson [8]).

Remark 4.4. In Example 1, $u^{\alpha}(\lambda)$ had the inverse Laplace transform $\xi_{\alpha}(d x)$ in the space of signed measures. Further it is seen that the transition function $P(t, x, d y)$ itself has the representation

$$
P(t, x, d y)=\sum_{k=0}^{\infty} e^{v_{h} t} \sum_{\alpha \in R_{k}} \phi_{\alpha}(x)\left\{\xi_{\alpha}\left(d y^{\prime}\right)+\sum_{\substack{\beta \in R_{k} \\ \beta<\alpha}} b_{\beta}^{\alpha}(t) \xi_{\beta}(d y)\right\}
$$

(strictly speaking $b_{\beta}^{\alpha}(t)=0$ in Example 1). But this is not valid for Example 2. We can prove that if $P_{e_{i}}\left(x_{t}=0\right)>0$ there is a $u^{j}(\lambda)$ having the inverse Laplace transform $\xi^{j}(d x)$ with $\xi^{j}(E) \geqq 0, E \subset R_{+}^{d}-\{0\}$ and $\xi^{j}(\{0\}) \leqq 0$.

\section{References}

[1] Coddington, E. A. and Levinson, N., Theory of Ordinary Differential Equations, Mcgraw-Hill, New York, 1955.

[2] Gantmacher, F. R., The Theory of Matrices, I, II, Chelsea, New York, 1959 (English translation).

[3] Jirina, M., Stochastic branching processes with continuous state space, Cizechoslovak Math. J. 8 (1958), 292-312.

[4] Karlin, S. and McGregor, J., Spectral theory of branching processes, I, II, Z. Wahrsheinlichkeitstheorie verw. Geb. 5 (1966), 6-33; 34-54.

[5] — - On the spectral representation of branching processes with mean one, J. Math. Anal. Appl. 21 (1968), 485-495.

[6] —-, Iteration of analytic function of several variables: Problems in Analysis - A Symposium in Honor of Salomon Bochner, Princeton U. P., 1970, 81-92.

[7] Kotelyanskii, D. M., On some properties of matrices with positive elements, Math. Sb. 31 (1952), 497-506 (Russian).

[8] Nelson, E., The adjoint Markov process, Dvke Math. J. 25 (1958), 671-690.

[9] Ogura, Y., Spectral representation for branching processes on the real half line, Publ. RIMS, Kyoto Univ. 5 (1970), 423-441. 
[10] Poincarć, H., Sur les propriétés des fonctions définies par lcs ćquations aux différences partielles, Oeuvres, 1, 36-129.

[11] Sevestyanov, B. A., The theory of branching random processes, Uspehi Matemat. Nauk 6 (1951), 47-99 (Russian).

[12] Sternberg, S., Local contractions and a theorcm of Poincaré, Amcr. J. Math. 79 (1957), 809-824.

[13] Watanabe, S., On two dimensional Markov processes with branching property, Transactions Amer. Math. Soc. 136 (1969), 447-466. 
